\title{
Increasing the Fault Coverage of a Truncated Test Set
}

\author{
IRITH POMERANZ, Purdue University
}

\begin{abstract}
Defect-aware, cell-aware, and gate-exhaustive faults are described by input patterns of subcircuits or cells that are expected to activate defects. Even with single-cycle faults, an $n$-input subcircuit can have up to $2^{n}$ faults with unique fault detection conditions, resulting in a large test set. Such a test set may have to be truncated to fit in the tester memory or satisfy constraints on test application time. In this case, a loss of fault coverage is inevitable. This article considers the test set denoted by $T_{1}$ obtained after truncating a larger test set denoted by $T_{0}$. Suppose that the truncation reduces the set of detected faults from the set denoted by $D_{0}$ to the set denoted by $D_{1}$. The procedure described in this article modifies the tests in $T_{1}$ to gain the detection of faults from $D_{0} \backslash D_{1}$, even at the cost of losing the detection of faults from $D_{1}$. The goal is to reduce the fault coverage loss by computing a test set denoted by $T_{2}$ that detects a set of faults denoted by $D_{2}$ such that $\left|T_{2}\right|=\left|T_{1}\right|$ and $\left|D_{2}\right|>\left|D_{1}\right|$. Experimental results for benchmark circuits demonstrate the ability of the procedure to increase the coverage of gate-exhaustive faults over several iterations.
\end{abstract}

\section{CCS Concepts: • Hardware $\rightarrow$ Test-pattern generation and fault simulation;}

Additional Key Words and Phrases: Gate-exhaustive faults, test compaction, test generation, stuck-at faults

ACM Reference format:

Irith Pomeranz. 2022. Increasing the Fault Coverage of a Truncated Test Set. ACM Trans. Des. Autom. Electron. Syst. 27, 6, Article 54 (June 2022), 16 pages.

https://doi.org/10.1145/3508459

\section{INTRODUCTION}

New technologies give rise to new types of defects [1-7] that can be modeled as defect-aware $[8,9]$, cell-aware [10-14] or gate-exhaustive [15-19] faults. These types of faults are described by input patterns of subcircuits or cells. The input patterns are such that they are expected to activate defects. In the case of defect-aware and cell-aware faults, the input patterns are selected by analyzing the layout of the subcircuit. In the case of gate-exhaustive faults, all of the input patterns are considered important. A fault associated with an input pattern $p_{i}$ of a subcircuit $G_{i}$ is detected by assigning $p_{i}$ to the inputs of the gate and propagating a fault effect from the output of $G_{i}$ to an observable output.

Even with single-cycle faults, an $n$-input subcircuit can have up to $2^{n}$ faults. The faults have unique detection conditions since they are associated with different input patterns of the subcircuit. As a result, a large test set may be obtained. In this case, the test set may have to be truncated to fit in the tester memory or satisfy constraints on the test application time. Before truncation,

This work was supported in part by SRC grant 2020-CT-2967.

Authors' address: I. Pomeranz, School of Electrical and Computer Engineering, Purdue University, West Lafayette, IN 47907 USA; email: pomeranz@ecn.purdue.edu.

Permission to make digital or hard copies of all or part of this work for personal or classroom use is granted without fee provided that copies are not made or distributed for profit or commercial advantage and that copies bear this notice and the full citation on the first page. Copyrights for components of this work owned by others than ACM must be honored. Abstracting with credit is permitted. To copy otherwise, or republish, to post on servers or to redistribute to lists, requires prior specific permission and/or a fee. Request permissions from permissions@acm.org.

(C) 2022 Association for Computing Machinery.

1084-4309/2022/06-ART54 \$15.00

https://doi.org/10.1145/3508459 


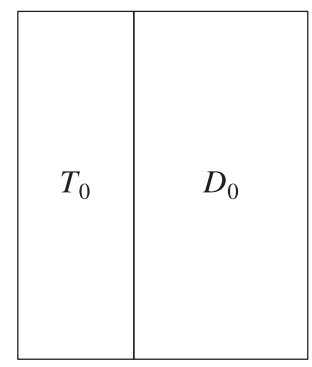

(a) $T_{0}$

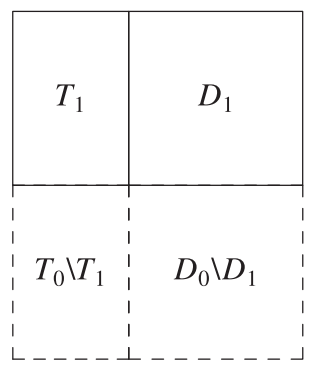

(b) $T_{1}$

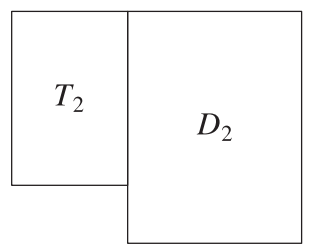

(c) $T_{2}$

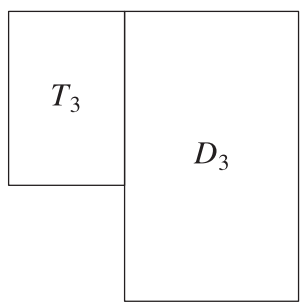

(d) $T_{3}$

Fig. 1. Test set truncation.

a test generation procedure may achieve complete or close-to-complete fault coverage for the fault model(s) of interest. However, the number of tests in the test set may be excessive even if test compaction is used [20-23]. It is then necessary to truncate the test set. Truncation reduces the number of tests and, along with it, the fault coverage. As a result, the fault coverage after truncation is not complete. The procedures described in $[24,25]$ reorder a test set before it is truncated to steepen its fault coverage curve. With a steeper fault coverage curve, a higher fault coverage is obtained after truncation. However, a loss of fault coverage is inevitable when a test set is truncated.

This article considers the test set obtained after truncation. Suppose that a test set $T_{0}$ is truncated into a test set $T_{1}$. Suppose further that the set of detected faults is reduced from $D_{0}$ to $D_{1}$. This is illustrated by Figures 1(a) and 1(b). With the process from [24, 25], $T_{1} \subset T_{0}$. Therefore, $D_{1}$ is limited by the tests included in $T_{0}$. To remove this limitation, the procedure described in this article modifies the tests in $T_{1}$ to gain the detection of faults from $D_{0} \backslash D_{1}$, even at the cost of losing the detection of faults from $D_{1}$. The goal of the procedure is to obtain a test set $T_{2}$ that detects a set of faults $D_{2}$ such that $\left|T_{2}\right|=\left|T_{1}\right|$ and $\left|D_{2}\right|>\left|D_{1}\right|$. This is illustrated by Figure 1(c). Thus, the goal is to reduce the fault coverage loss that occurred when the test set was truncated by modifying the tests. In an iterative process, the procedure produces test sets $T_{k}$, for $k \geq 2$, such that $\left|T_{k}\right|=\left|T_{k-1}\right|$ and $\left|D_{k}\right|>$ $\left|D_{k-1}\right|$, with reduced fault coverage loss. Figure 1(d) shows the test set $T_{3}$ and the set of detected faults $D_{3}$. Since the number of tests in $T_{0}$ is determined by a test-generation procedure, and the number of tests in $T_{k}$, for $k \geq 1$, is determined by constraints on the tester memory or test application time, the procedure described in this article does not attempt to change the number of tests in $T_{k}$.

The procedure is applied to a compact test set for single stuck-at faults and single-cycle gateexhaustive faults. Truncation is performed after the test set is reordered using a procedure similar to the one from [25]. Truncation is done such that the stuck-at fault coverage is maintained. However, the single-cycle gate-exhaustive fault coverage decreases inevitably when the number of tests is reduced. The procedure described in this article is applied to increase the single-cycle gate-exhaustive fault coverage of the truncated test set.

Gate-exhaustive faults are interesting for this study since the number of faults is large, and many of them are undetectable. In addition, there are large variations among different circuits in the percentage of tests that need to be added to a stuck-at test set to detect gate-exhaustive faults. Since it is not known in advance how many tests will be needed, tests are generated for all of the faults. Truncation may then remove different percentages of tests for different circuits and result in a different loss of fault coverage for different circuits. This allows the procedure described in this article to be studied over a large range of truncated test sets. The same procedure is applicable with other fault models and when several different fault models are targeted [26-30]. To demonstrate the broader applicability of the procedure, it is applied to a randomly selected subset of all of the 
gate-exhaustive faults. Cell-aware faults also constitute a subset of gate-exhaustive faults when the same cells or gates are used.

For further illustration, the procedure is applied to a compressed launch-on-shift test set that targets single stuck-at, transition, single-cycle, and two-cycle gate-exhaustive faults. In this case, truncation is performed such that it maintains the stuck-at and transition fault coverage. However, the coverage of gate-exhaustive faults of both types may be reduced. For the description of the algorithm developed in this article, single-cycle tests for stuck-at and single-cycle gate-exhaustive faults are considered as a concrete example. Compressed two-cycle tests for four fault models are considered later.

The article is organized as follows. Section 2 describes the problem addressed in this article. Section 3 provides an overview of the procedure used for solving it. Section 4 provides details of its implementation. Section 5 presents experimental results for benchmark circuits considering single-cycle tests. Section 6 discusses experimental results for compressed two-cycle tests. Section 7 presents concluding remarks.

\section{PROBLEM FORMULATION}

The procedure described in this article accepts a test set $T_{0}$ that detects a set of faults $D_{0}$. The set $D_{0}$ consists of single-cycle gate-exhaustive faults as well as single stuck-at faults. The procedure truncates $T_{0}$ to obtain the test set $T_{1}$ and then modifies $T_{1}$ to obtain the test set $T_{2}$. Truncation of $T_{1}$ and modification of $T_{2}$ are performed such that $T_{1}$, and then $T_{2}$, detect all of the single stuckat faults in $D_{0}$. This is justified by the fact that complete coverage of single stuck-at faults is a minimum requirement for testing a circuit.

Fault simulation with fault dropping of $D_{0}$ under $T_{0}$ yields, for every test $t_{i} \in T_{0}$, a set of detected stuck-at faults denoted by $D_{s a}\left(t_{i}\right)$, and a set of detected gate-exhaustive faults denoted by $D_{g e}\left(t_{i}\right)$. Before truncating $T_{0}$, its tests are ordered from high to low size of $D_{s a}\left(t_{i}\right)$. For the same size of $D_{s a}\left(t_{i}\right)$, the tests are ordered from high to low size of $D_{g e}\left(t_{i}\right)$. With this order, the first tests in $T_{0}$ detect all of the single stuck-at faults. The remaining tests detect only gate-exhaustive faults. In both cases, the tests are ordered such that tests appearing earlier detect more faults.

After reordering the tests, fault simulation is carried out again to update the sets $D_{s a}\left(t_{i}\right)$ and $D_{g e}\left(t_{i}\right)$. Reordering and fault simulation are repeated until no further changes to the order of the tests are obtained.

Considering the ordered test set $T_{0}$, and a parameter $0<\theta<\left|T_{0}\right|$, a truncated test set $T_{1}$ includes the first $\theta$ tests from $T_{0}$. Fault simulation with fault dropping of $D_{0}$ under $T_{1}$ yields a set $D_{1} \subset D_{0}$ of detected faults. The value of $\theta$ is selected such that $D_{1}$ includes all the single stuck-at faults from $D_{0}$.

Figures $1(\mathrm{a})$ and (b) demonstrate the truncation of $T_{0}$ into $T_{1}$. The test set $T_{1}$ excludes the tests in $T_{0} \backslash T_{1}$ to reduce the number of tests. As a result, it loses the detection of the faults in $D_{0} \backslash D_{1}$. Reordering of $T_{0}$ ensures that $T_{1}$ loses the detection of as few faults as possible. However, a loss in fault coverage is inevitable when the test set is truncated. In addition, the selection of $T_{1}$ is performed without modifying the tests in $T_{0}$. Even though $T_{0}$ is optimized for the detection of $D_{0}$, the truncated test set $T_{1}$ may not be optimized for the detection of $D_{1}$. Thus, with the same number of tests, it may be possible to detect more faults if different tests are used.

The possibility of detecting more faults is illustrated by Figure 1(c). The test set $T_{2}$ has the same size as $T_{1}$. It detects a set of faults $D_{2} \subset D_{0}$ that is larger than $D_{1}$. The computation of $T_{2}$ is the goal of the procedure described in this article. The same procedure is applied iteratively to produce test sets $T_{3}, T_{4}, \ldots$ with increased sets of detected faults. Figure $1(\mathrm{~d})$ illustrates the case of $T_{3}$.

Problem Formulation: Given a test set $T_{0}$ that detects a set of faults $D_{0}$, and a truncated test set $T_{1}$ that detects a set of faults $D_{1} \subset D_{0}$, compute a test set $T_{2}$ with a set of detected faults $D_{2} \subseteq D_{0}$ such that $\left|T_{2}\right|=\left|T_{1}\right|$ and $\left|D_{2}\right|>\left|D_{1}\right|$. 


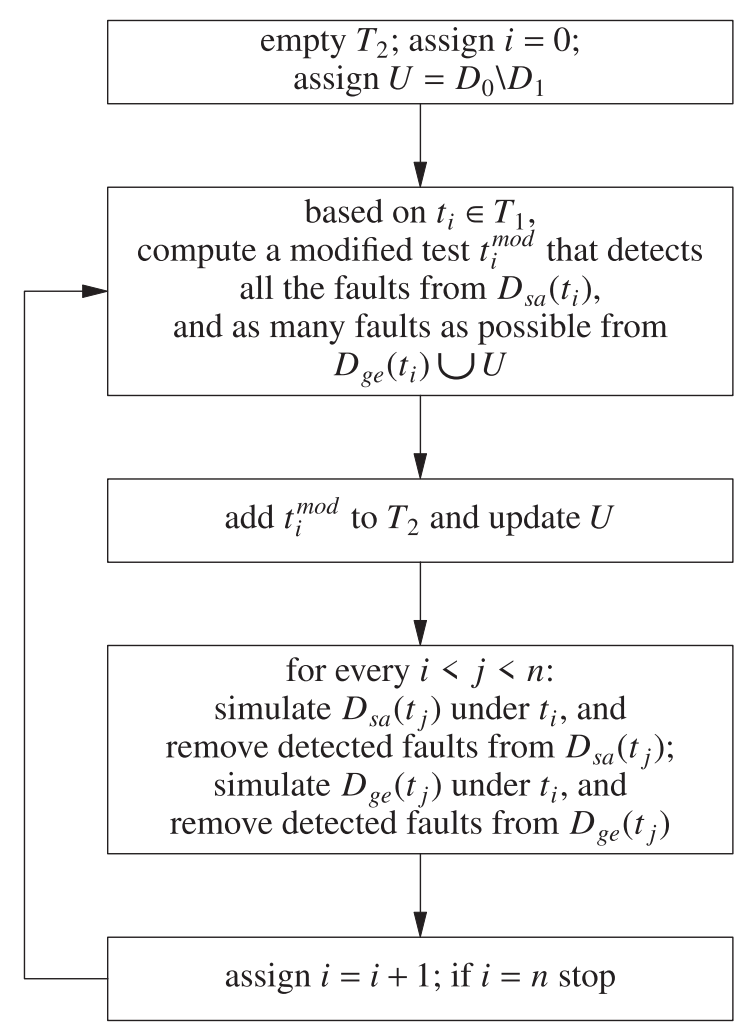

Fig. 2. Procedure for increasing the fault coverage after truncation.

\section{PROCEDURE OVERVIEW}

The procedure described in this article uses the gate-exhaustive faults in $D_{0} \backslash D_{1}$ as targets for the modification of the tests in $T_{1}$. The goal is to increase the numbers of gate-exhaustive faults the tests in $T_{1}$ detect. In this process, the procedure accepts losing the detection of gate-exhaustive faults from $D_{1}$ if it can detect gate-exhaustive faults from $D_{0} \backslash D_{1}$ in their place such that the number of detected gate-exhaustive faults is increased. This proceeds as illustrated by Figure 2 and described next.

\subsection{Procedure}

Let $T_{1}=\left\{t_{0}, t_{1}, \ldots, t_{n-1}\right\}$. Fault simulation with fault dropping yields sets of detected faults $D_{s a}\left(t_{i}\right)$ and $D_{g e}\left(t_{i}\right)$ for every test $t_{i} \in T_{1}$. The procedure constructs a test set $T_{2}$ by considering the tests from $T_{1}$ one by one. Every test is modified before it is added to $T_{2}$, as described next.

Initially, $T_{2}=\emptyset$, and $U=D_{0} \backslash D_{1}$ includes the gate-exhaustive faults that are detected by $T_{0}$ but not by $T_{1}$. When a test $t_{i} \in T_{1}$ is considered, the goal of the procedure is to compute a new test $t_{i}^{\text {mod }}$ that satisfies the following conditions.

(1) $t_{i}^{\text {mod }}$ detects all of the faults from $D_{s a}\left(t_{i}\right)$. This is important for ensuring that all of the single stuck-at faults detected by $T_{0}$ (and $T_{1}$ ) are also detected by $T_{2}$.

(2) $t_{i}^{\text {mod }}$ detects as many faults as possible from the set $D_{g e}\left(t_{i}\right) \cup U$. This condition allows the procedure to substitute gate-exhaustive faults in $D_{1}$ with gate-exhaustive faults in $U$ if this results in an increase in the gate-exhaustive fault coverage of $T_{2}$. Without modification, $t_{i}^{\text {mod }}=t_{i}$ detects 
the faults in $D_{g e}\left(t_{i}\right)$. Therefore, the minimum number of gate-exhaustive faults that $t_{i}^{\text {mod }}$ will detect is $\left|D_{g e}\left(t_{i}\right)\right|$. By optimizing $t_{i}^{\text {mod }}$, the procedure may increase the number of detected gateexhaustive faults, thus increasing the gate-exhaustive fault coverage of $T_{2}$ relative to $T_{1}$.

The computation of $t_{i}^{\text {mod }}$ can be done by a test generation procedure targeting the faults in $D_{s a}\left(t_{i}\right) \cup D_{g e}\left(t_{i}\right) \cup U$. A different implementation is described in Section 4.

After computing $t_{i}^{\text {mod }}$, the procedure includes it in $T_{2}$. It updates $U$ to include gate-exhaustive faults from $D_{g e}\left(t_{i}\right)$ whose detection was lost, and exclude new gate-exhaustive faults that $t_{i}^{\text {mod }}$ detects. The updated set is $\left(U \cup D_{g e}\left(t_{i}\right)\right) \backslash D_{g e}\left(t_{i}^{\text {mod }}\right)$.

Next, for $i<j<n$, the procedure simulates the faults in $D_{s a}\left(t_{j}\right) \cup D_{g e}\left(t_{j}\right)$ under $t_{i}^{\text {mod }}$. If a fault $f \in D_{s a}\left(t_{j}\right)$ is detected by $t_{i}^{\text {mod }}$, the procedure removes the fault from $D_{s a}\left(t_{j}\right)$. Similarly, if a fault $f \in D_{g e}\left(t_{j}\right)$ is detected by $t_{i}^{\text {mod }}$, the procedure removes the fault from $D_{g e}\left(t_{j}\right)$. This is important since having fewer faults in $D_{s a}\left(t_{j}\right)$ and $D_{g e}\left(t_{j}\right)$ ensures that $t_{j}$ is easier to modify later.

Experimental results indicate that a test $t_{i}$ close to the beginning of $T_{1}$ cannot be modified to increase $D_{g e}\left(t_{i}\right)$ significantly. The reason is that the sets $D_{s a}\left(t_{i}\right)$ and $D_{g e}\left(t_{i}\right)$ are large for tests that appear at the beginning of the test set, and the flexibility to modify them is low. To avoid the computational effort of attempting to modify tests that cannot be modified, the procedure attempts to modify tests starting from $t_{m / 4}$, where $m$ is the number of tests for stuck-at faults in $T_{1}$. The procedure copies $t_{0}, \ldots, t_{m / 4-1}$ from $T_{1}$ to $T_{2}$ unmodified. It attempts to modify the tests $t_{m / 4}, \ldots, t_{n-1}$.

Using the same process for $k>2$, the procedure uses $T_{k-1}$ to compute a modified test set $T_{k}$ with an increased gate-exhaustive fault coverage. This continues until the gate-exhaustive fault coverage of $T_{k}$ is equal to that of $T_{k-1}$.

\subsection{Example}

To illustrate the procedure, benchmark circuit $s 1423$ is considered in Table 1 . The test set $T_{0}$ contains 118 tests, including 38 tests for stuck-at faults. The test set detects 1653 gate-exhaustive faults. It is truncated into a test set that contains 76 tests and detects 1599 gate-exhaustive faults. The procedure considers the tests $t_{9}, t_{10}, \ldots, t_{75}$. Some of these tests are shown in Table 1 . The first column of Table 1 shows the index of a test $t_{i}$. This is followed by the number of gate-exhaustive faults detected by $t_{i}$. Column init shows the number of faults detected by $t_{i}$ initially, when $t_{i}$ is first considered. Column modify shows the number of faults detected by $t_{i}$ after it is modified. Column move shows the number of faults detected by $t_{i}$ after the procedure simulates under $t_{i}$ faults that are detected by tests appearing later than $t_{i}$. This moves detected faults from later tests to $t_{i}$. Column from shows the indices of the tests from which faults are moved to $t_{i}$.

When $t_{9}$ is considered, the procedure finds that it detects 38 gate-exhaustive faults. Modifying $t_{9}$ does not result in the detection of new faults, and no faults are moved from later tests.

When $t_{10}$ is considered, the procedure finds that it detects 43 gate-exhaustive faults. Modifying $t_{10}$ does not result in the detection of new faults. One fault is moved from $t_{18}$ to $t_{10}$. Later, when $t_{18}$ is considered, this allows the procedure to modify it such that it detects one new fault.

Five faults are moved from $t_{21}$ to tests that appear earlier, one to each of $t_{11}, t_{12}$, and $t_{17}$, and two to $t_{13}$. When $t_{21}$ is considered, the procedure finds that it detects four gate-exhaustive faults. Modifying $t_{21}$ results in the detection of one new fault, and four faults are moved from later tests to $t_{21}$.

Additional examples are shown in Table 1. After the construction of $T_{2}$ is complete, the test set detects 1629 gate-exhaustive faults.

It is also interesting to consider the subsets of gate-exhaustive faults detected by a test $t_{i}$ before and after it is modified. The test $t_{65}$ of $s 1423$ is considered in Table 2 . Before the test is modified, 
Table 1. Example of Computing $T_{2}$

\begin{tabular}{c|cccc}
\hline$i$ & init & modify & move & from \\
\hline 9 & 38 & 38 & 38 & \\
10 & 43 & 43 & 44 & $(18)$ \\
11 & 31 & 31 & 32 & $(21)$ \\
12 & 42 & 42 & 43 & $(21)$ \\
13 & 24 & 26 & 28 & $(21)$ \\
14 & 35 & 35 & 35 & \\
15 & 21 & 21 & 21 & \\
16 & 21 & 21 & 21 & \\
17 & 19 & 19 & 22 & $(21,27,41)$ \\
18 & 17 & 18 & 18 & \\
19 & 15 & 17 & 17 & \\
20 & 12 & 12 & 12 & \\
21 & 4 & 5 & 9 & $(22,42,50,66)$ \\
22 & 10 & 12 & 13 & $(34)$ \\
\hline 29 & 4 & 7 & 8 & $(46)$ \\
30 & 3 & 4 & 4 & \\
33 & 5 & 7 & 7 & \\
35 & 3 & 4 & 4 & \\
36 & 2 & 3 & 3 & \\
37 & 1 & 3 & 3 & \\
42 & 7 & 8 & 8 & \\
49 & 4 & 5 & 6 & $(57)$ \\
50 & 2 & 3 & 3 & \\
\hline
\end{tabular}

Table 2. Example of Detected Faults

\begin{tabular}{c|c|cccc}
\hline$i$ & step & \multicolumn{4}{|c}{ detected } \\
\hline 65 & init & $(689,10011011)$ & $(706,10001)$ & $(986,00011)$ & \\
& modify & $(689,10011011)$ & $(706,10001)$ & $(986,00101)$ & $(1421,10011)$ \\
\hline
\end{tabular}

it detects three faults. After it is modified, it detects four faults. The faults are shown in Table 2 in the format $\left(G_{a}, p_{b}\right)$, where $G_{a}$ is the index of a gate, and $p_{b}$ is the input pattern assigned to the gate inputs to activate the fault.

In the case of $t_{65}$, to allow a new fault, $(1421,10011)$, to be detected, the fault $(986,00011)$ is replaced with the fault $(986,00101)$.

\section{TEST MODIFICATION}

This section describes the computation of a modified test $t_{i}^{\text {mod }}$ based on a test $t_{i}$ with subsets of detected faults $D_{s a}\left(t_{i}\right)$ and $D_{g e}\left(t_{i}\right)$. As before, the set of gate-exhaustive faults whose detection was lost by the truncation of the test set and not recovered is denoted by $U$. The advantage of the procedure described in this section over test generation that targets the faults in $D_{s a}\left(t_{i}\right) \cup D_{g e}\left(t_{i}\right) \cup$ $U$ is that it keeps $t_{i}^{\text {mod }}$ as close as possible to $t_{i}$. In particular, it does not lose the detection of any fault from $D_{s a}\left(t_{i}\right)$ and, thus, does not need to target these faults again. In addition, it does not lose the detection of a fault from $D_{g e}\left(t_{i}\right)$ unless it can detect a different gate-exhaustive fault in its place. Consequently, $t_{i}^{\text {mod }}$ detects as many faults from $D_{g e}\left(t_{i}\right)$ as possible. 
The procedure starts with $t_{i}^{\text {mod }}=t_{i}$. It modifies $t_{i}^{\text {mod }}$ by complementing its bits one by one. After bit $b$ is complemented, let the test $t_{i, b}^{\text {mod }}$ be obtained. To decide whether the complementation of bit $b$ is acceptable, the procedure performs fault simulation as follows.

(1) The procedure simulates $D_{s a}\left(t_{i}^{\text {mod }}\right)$ under $t_{i, b}^{\text {mod }}$. If any fault is not detected, the complementation of bit $b$ is not acceptable. This condition ensures that all of the single stuck-at faults detected by $T_{0}$ (and $T_{1}$ ) are also detected by $T_{2}$.

(2) The procedure simulates $D_{g e}\left(t_{i}^{\text {mod }}\right) \cup U$ under $t_{i, b}^{\text {mod }}$. Let the subset of detected faults be $D_{g e}\left(t_{i, b}^{\text {mod }}\right)$. If $\left|D_{g e}\left(t_{i, b}^{\text {mod }}\right)\right|<\left|D_{g e}\left(t_{i}^{\text {mod }}\right)\right|$, the complementation of bit $b$ is not acceptable. This condition ensures that the number of detected gate-exhaustive faults is not decreased by the modification.

If both checks indicate that $t_{i, b}^{\text {mod }}$ is acceptable, the procedure assigns $t_{i}^{\text {mod }}=t_{i, b}^{\text {mod }}$. Faults in $D_{g e}\left(t_{i, b}^{\text {mod }}\right) \cap U$ are removed from $U$, and faults in $D_{g e}\left(t_{i}^{\text {mod }}\right) \backslash D_{g e}\left(t_{i, b}^{\text {mod }}\right)$ are added to $U$ to keep the set $U$ up-to-date. The set $U$ is updated by assigning $U=\left(U \cup D_{g e}\left(t_{i}^{\text {mod }}\right)\right) \backslash D_{g e}\left(t_{i, b}^{\text {mod }}\right)$.

All of the bits of $t_{i}^{\text {mod }}$ are considered a constant number of times. The constant four is used for the experiments. This is based on the experimental observation that fewer than four passes over the bits of a test are typically needed before no additional improvements in the subsets of detected faults can be achieved.

\section{EXPERIMENTAL RESULTS FOR SINGLE-CYCLE TESTS}

This section presents the results of applying the procedure for increasing the fault coverage of a truncated test set to benchmark circuits using single-cycle tests for stuck-at and single-cycle gate-exhaustive faults.

The set of gate-exhaustive faults is the one computed in [19]. In addition, in a separate experiment, a quarter of the gate-exhaustive faults from [19] are selected randomly and used as target faults. This demonstrates a case that is closer to the case in which cell-aware faults are used. In the case of cell-aware faults, assuming the same cells or gates, a subset of gate-exhaustive faults are identified as important for every gate; only these faults are included in the set of target faults.

The test set $T_{0}$ consists of a compact test set for single stuck-at faults, topped off with tests for gate-exhaustive faults from [19]. Only tests that detect additional gate-exhaustive faults are included in $T_{0}$. The set $D_{0}$ includes all of the stuck-at and gate-exhaustive faults detected by $T_{0}$.

The set of detectable gate-exhaustive faults in $D_{0}$ is taken as the universe of gate-exhaustive faults for the computation of a percentage of detected faults, which is referred to as the gateexhaustive fault efficiency. The test set is reordered and then truncated as described in Section 2 using a parameter $0<\theta<\left|T_{0}\right|$. Values of $\theta$ are selected as follows.

Let $\theta_{0}$ be the number of tests in a compact test set for single stuck-at faults. After reordering, these tests appear at the beginning of $T_{0}$. For $\mu=2$ and 3 , the procedure is applied with $\theta=\mu \theta_{0}$. The rationale for this selection is as follows.

With $\theta=2 \theta_{0}$, the number of additional tests available for detecting gate-exhaustive faults is the same as the number required for detecting single stuck-at faults. The case in which $\theta=3 \theta_{0}$ illustrates the situation in which a larger test set can be accommodated.

For some benchmark circuits, $\theta=2 \theta_{0}$ results in the removal of a large percentage of tests from $T_{0}$. For other circuits, the percentage of tests removed is smaller. The use of $\theta=3 \theta_{0}$ results in lower percentages of tests being removed from $T_{0}$. The percentage of tests removed determines the fault coverage loss for gate-exhaustive faults. Overall, the use of $\theta=2 \theta_{0}$ and $3 \theta_{0}$, and the selection of gate-exhaustive faults, result in a large range of different truncated test sets, with different fault coverages, that bring out the ability of the procedure described in this article to increase the fault coverage in different situations. 
Table 3. Experimental Results $\left|T_{1}\right| /\left|T_{0}\right|<35 \%$

\begin{tabular}{|c|c|c|c|c|c|c|c|c|c|}
\hline circuit & inp & $\mu$ & $k$ & tests & \%tests & s.a. & g.exh & gap & ntime \\
\hline s38417 & 1664 & 2 & 1 & 206 & 12.61 & 99.680 & 80.784 & 0.000 & 12.58 \\
\hline s38417 & 1664 & 2 & 2 & 206 & 12.61 & 99.680 & 85.846 & 26.343 & 811.73 \\
\hline s38417 & 1664 & 2 & 50 & 206 & 12.61 & 99.680 & 88.409 & 39.682 & 43091.31 \\
\hline s38417 & 1664 & $\mathrm{~s} 2$ & 1 & 206 & 19.25 & 99.680 & 81.811 & 0.000 & 11.52 \\
\hline s38417 & 1664 & $\mathrm{~s} 2$ & 2 & 206 & 19.25 & 99.680 & 91.297 & 52.153 & 603.25 \\
\hline s38417 & 1664 & $\mathrm{~s} 2$ & 28 & 206 & 19.25 & 99.680 & 94.916 & 72.050 & 19760.49 \\
\hline b20 & 527 & 2 & 1 & 852 & 20.86 & 95.586 & 90.162 & 0.000 & 17.94 \\
\hline b20 & 527 & 2 & 2 & 852 & 20.86 & 95.766 & 90.871 & 7.200 & 270.33 \\
\hline b20 & 527 & 2 & 23 & 852 & 20.86 & 95.807 & 91.341 & 11.983 & 5702.19 \\
\hline s15850 & 611 & 2 & 1 & 236 & 21.55 & 97.511 & 83.332 & 0.000 & 9.20 \\
\hline s15850 & 611 & 2 & 2 & 236 & 21.55 & 97.511 & 85.618 & 13.717 & 339.24 \\
\hline s15850 & 611 & 2 & 32 & 236 & 21.55 & 97.511 & 88.705 & 32.237 & 10060.41 \\
\hline simple_spi & 146 & 2 & 1 & 72 & 23.38 & 100.000 & 67.806 & 0.000 & 5.19 \\
\hline simple_spi & 146 & 2 & 2 & 72 & 23.38 & 100.000 & 74.063 & 19.434 & 76.12 \\
\hline simple_spi & 146 & 2 & 7 & 72 & 23.38 & 100.000 & 74.747 & 21.558 & 432.94 \\
\hline b14 & 280 & 2 & 1 & 706 & 25.35 & 95.966 & 86.763 & 0.000 & 10.65 \\
\hline b14 & 280 & 2 & 2 & 706 & 25.35 & 96.193 & 86.955 & 1.455 & 205.40 \\
\hline b14 & 280 & 2 & 6 & 706 & 25.35 & 96.205 & 87.128 & 2.764 & 984.46 \\
\hline s9234 & 247 & 2 & 1 & 286 & 28.66 & 93.946 & 85.674 & 0.000 & 13.72 \\
\hline s9234 & 247 & 2 & 2 & 286 & 28.66 & 93.946 & 87.703 & 14.167 & 192.38 \\
\hline s9234 & 247 & 2 & 14 & 286 & 28.66 & 93.946 & 88.819 & 21.955 & 2477.91 \\
\hline b20 & 527 & 3 & 1 & 1278 & 31.29 & 95.586 & 92.650 & 0.000 & 16.97 \\
\hline b20 & 527 & 3 & 2 & 1278 & 31.29 & 95.773 & 92.905 & 3.468 & 388.78 \\
\hline b20 & 527 & 3 & 13 & 1278 & 31.29 & 95.812 & 93.123 & 6.435 & 5310.94 \\
\hline s15850 & 611 & 3 & 1 & 354 & 32.33 & 97.511 & 90.083 & 0.000 & 9.32 \\
\hline s15850 & 611 & 3 & 2 & 354 & 32.33 & 97.511 & 91.042 & 9.673 & 528.24 \\
\hline s15850 & 611 & 3 & 53 & 354 & 32.33 & 97.511 & 93.833 & 37.818 & 26999.10 \\
\hline simple_spi & 146 & $\mathrm{~s} 2$ & 1 & 72 & 32.43 & 100.000 & 72.138 & 0.000 & 9.86 \\
\hline simple_spi & 146 & $\mathrm{~s} 2$ & 2 & 72 & 32.43 & 100.000 & 90.679 & 66.545 & 148.57 \\
\hline simple_spi & 146 & s2 & 6 & 72 & 32.43 & 100.000 & 93.820 & 77.818 & 818.14 \\
\hline i2c & 145 & 2 & 1 & 90 & 33.46 & 100.000 & 88.819 & 0.000 & 5.94 \\
\hline $\mathrm{i} 2 \mathrm{c}$ & 145 & 2 & 2 & 90 & 33.46 & 100.000 & 91.986 & 28.325 & 71.56 \\
\hline i2c & 145 & 2 & 12 & 90 & 33.46 & 100.000 & 92.812 & 35.714 & 822.56 \\
\hline sasc & 132 & 2 & 1 & 44 & 34.65 & 100.000 & 74.347 & 0.000 & 5.17 \\
\hline sasc & 132 & 2 & 2 & 44 & 34.65 & 100.000 & 79.323 & 19.396 & 135.33 \\
\hline sasc & 132 & 2 & 6 & 44 & 34.65 & 100.000 & 79.853 & 21.463 & 720.33 \\
\hline
\end{tabular}

The results are shown in Tables 3 to 7 as follows. The circuits are arranged from low to high value of $\left|T_{1}\right| /\left|T_{0}\right| \cdot 100$, which is the percentage of tests from $T_{0}$ included in $T_{1}$ after truncation. Each table has a different range of the percentage $\left|T_{1}\right| /\left|T_{0}\right| \cdot 100$.

There are several rows for every circuit, corresponding to $T_{1}, T_{2}$, and the final test set obtained by the procedure from Figure 2.

For every test set, after the circuit name, column inp shows the number of inputs. Column $\mu$ shows the value of this parameter. When only a quarter of the gate-exhaustive faults are considered, the value of $\mu$ is preceded by an "s." Column $k$ shows the iteration of the procedure. 
Table 4. Experimental Results 35\% $\leq\left|T_{1}\right| /\left|T_{0}\right|<45 \%$

\begin{tabular}{|c|c|c|c|c|c|c|c|c|c|}
\hline circuit & inp & $\mu$ & $k$ & tests & \%tests & s.a. & g.exh & gap & ntime \\
\hline simple_spi & 146 & 3 & 1 & 108 & 35.06 & 100.000 & 80.598 & 0.000 & 7.63 \\
\hline simple_spi & 146 & 3 & 2 & 108 & 35.06 & 100.000 & 85.436 & 24.935 & 163.88 \\
\hline simple_spi & 146 & 3 & 7 & 108 & 35.06 & 100.000 & 86.348 & 29.634 & 978.63 \\
\hline spi & 274 & 2 & 1 & 804 & 35.34 & 99.992 & 83.994 & 0.000 & 14.72 \\
\hline spi & 274 & 2 & 2 & 804 & 35.34 & 99.992 & 86.461 & 15.411 & 236.57 \\
\hline spi & 274 & 2 & 23 & 804 & 35.34 & 99.992 & 88.720 & 29.524 & 4549.54 \\
\hline wb_dma & 738 & 2 & 1 & 130 & 36.93 & 100.000 & 91.571 & 0.000 & 15.83 \\
\hline wb_dma & 738 & 2 & 2 & 130 & 36.93 & 100.000 & 95.179 & 42.814 & 765.61 \\
\hline wb_dma & 738 & 2 & 12 & 130 & 36.93 & 100.000 & 96.282 & 55.894 & 9745.93 \\
\hline b14 & 280 & 3 & 1 & 1059 & 38.03 & 95.966 & 89.029 & 0.000 & 10.74 \\
\hline b14 & 280 & 3 & 2 & 1059 & 38.03 & 96.211 & 89.241 & 1.931 & 299.23 \\
\hline b14 & 280 & 3 & 9 & 1059 & 38.03 & 96.228 & 89.324 & 2.692 & 2340.71 \\
\hline s15850 & 611 & s2 & 1 & 236 & 38.94 & 97.511 & 87.190 & 0.000 & 9.51 \\
\hline s15850 & 611 & s2 & 2 & 236 & 38.94 & 97.511 & 92.066 & 38.063 & 556.88 \\
\hline s15850 & 611 & s2 & 13 & 236 & 38.94 & 97.511 & 96.076 & 69.369 & 6684.05 \\
\hline systemcaes & 928 & 2 & 1 & 240 & 39.41 & 99.997 & 91.324 & 0.000 & 11.54 \\
\hline systemcaes & 928 & 2 & 2 & 240 & 39.41 & 99.997 & 92.465 & 13.147 & 445.55 \\
\hline systemcaes & 928 & 2 & 7 & 240 & 39.41 & 99.997 & 92.798 & 16.990 & 3251.41 \\
\hline s38584 & 1464 & 2 & 1 & 284 & 40.51 & 95.567 & 94.141 & 0.000 & 9.41 \\
\hline s38584 & 1464 & 2 & 2 & 284 & 40.51 & 95.567 & 96.558 & 41.261 & 1329.47 \\
\hline s38584 & 1464 & 2 & 20 & 284 & 40.51 & 95.567 & 97.379 & 55.266 & 31337.49 \\
\hline b15 & 483 & 2 & 1 & 850 & 41.04 & 98.938 & 88.247 & 0.000 & 14.82 \\
\hline b15 & 483 & 2 & 2 & 850 & 41.04 & 98.938 & 91.518 & 27.831 & 344.13 \\
\hline b15 & 483 & 2 & 24 & 850 & 41.04 & 98.938 & 92.840 & 39.083 & 7524.47 \\
\hline sasc & 132 & s2 & 1 & 44 & 42.72 & 100.000 & 79.608 & 0.000 & 6.75 \\
\hline sasc & 132 & $\mathrm{~s} 2$ & 2 & 44 & 42.72 & 100.000 & 98.042 & 90.400 & 125.00 \\
\hline sasc & 132 & s2 & 4 & 44 & 42.72 & 100.000 & 98.858 & 94.400 & 415.00 \\
\hline s9234 & 247 & 3 & 1 & 429 & 42.99 & 93.946 & 90.849 & 0.000 & 13.82 \\
\hline s9234 & 247 & 3 & 2 & 429 & 42.99 & 93.946 & 91.988 & 12.451 & 314.57 \\
\hline s9234 & 247 & 3 & 12 & 429 & 42.99 & 93.946 & 92.463 & 17.639 & 2815.32 \\
\hline systemcaes & 928 & s2 & 1 & 240 & 43.56 & 99.997 & 92.152 & 0.000 & 13.49 \\
\hline systemcaes & 928 & s2 & 2 & 240 & 43.56 & 99.997 & 98.498 & 80.859 & 255.73 \\
\hline systemcaes & 928 & s2 & 8 & 240 & 43.56 & 99.997 & 99.495 & 93.560 & 2781.03 \\
\hline
\end{tabular}

Column tests shows the number of tests in $T_{k}$. This number does not change with $k$. Column $\%$ tests shows the number of tests in $T_{k}$ as a percentage of the number of tests in $T_{0},\left|T_{k}\right| /\left|T_{0}\right| \cdot 100$.

Column s.a. shows the stuck-at fault coverage. Column g.exh shows the gate-exhaustive fault efficiency. Column gap measures the improvement in the gate-exhaustive fault efficiency as $\frac{\left|D_{k}\right|-\left|D_{1}\right|}{\left|D_{0}\right|-\left|D_{1}\right|} \cdot 100 \%$. The denominator is the gap between $D_{1}$ and $D_{0}$. The numerator is the extent to which the gap is covered by $T_{k}$. The ratio is the percentage improvement in the gate-exhaustive fault efficiency gap. This metric has a range of $0 \%$ to $100 \%$ even when the fault efficiency achieved by $D_{1}$ is high, and the maximum improvement possible in the fault efficiency is low. For example, when the fault efficiency is increased by $1 \%$ from $50 \%$ to $51 \%$, the improvement in the gap is $(51-50) /(100-50) \cdot 100=2 \%$. When the fault efficiency is increased by $1 \%$ from $90 \%$ to $91 \%$, the improvement in the gap is $(91-90) /(100-90) \cdot 100=10 \%$. 
Table 5. Experimental Results $45 \% \leq\left|T_{1}\right| /\left|T_{0}\right|<55 \%$

\begin{tabular}{|c|c|c|c|c|c|c|c|c|c|}
\hline circuit & inp & $\mu$ & $k$ & tests & \%tests & s.a. & g.exh & gap & ntime \\
\hline des_area & 367 & 2 & 1 & 232 & 45.05 & 100.000 & 84.062 & 0.000 & 25.05 \\
\hline des_area & 367 & 2 & 2 & 232 & 45.05 & 100.000 & 89.429 & 33.677 & 316.13 \\
\hline des_area & 367 & 2 & 28 & 232 & 45.05 & 100.000 & 92.541 & 53.202 & 9420.62 \\
\hline s5378 & 214 & 2 & 1 & 222 & 45.12 & 98.867 & 86.650 & 0.000 & 10.63 \\
\hline s5378 & 214 & 2 & 2 & 222 & 45.12 & 98.867 & 91.894 & 39.277 & 178.22 \\
\hline s5378 & 214 & 2 & 21 & 222 & 45.12 & 98.867 & 93.496 & 51.282 & 3543.05 \\
\hline b20 & 527 & s2 & 1 & 852 & 47.05 & 95.586 & 90.961 & 0.000 & 17.85 \\
\hline b20 & 527 & s2 & 2 & 852 & 47.05 & 95.963 & 92.618 & 18.330 & 517.56 \\
\hline b20 & 527 & s2 & 21 & 852 & 47.05 & 96.049 & 94.029 & 33.948 & 9696.21 \\
\hline des_area & 367 & s2 & 1 & 234 & 47.46 & 100.000 & 87.078 & 0.000 & 9.56 \\
\hline des_area & 367 & s2 & 2 & 234 & 47.46 & 100.000 & 98.685 & 89.822 & 162.72 \\
\hline des_area & 367 & s2 & 9 & 234 & 47.46 & 100.000 & 99.954 & 99.645 & 1465.40 \\
\hline wb_dma & 738 & s2 & 1 & 132 & 47.65 & 100.000 & 92.385 & 0.000 & 8.28 \\
\hline wb_dma & 738 & s2 & 2 & 132 & 47.65 & 100.000 & 99.077 & 87.879 & 442.68 \\
\hline wb_dma & 738 & s2 & 6 & 132 & 47.65 & 100.000 & 99.667 & 95.623 & 2931.31 \\
\hline s38584 & 1464 & s2 & 1 & 284 & 48.71 & 95.567 & 94.921 & 0.000 & 9.50 \\
\hline s38584 & 1464 & s2 & 2 & 284 & 48.71 & 95.567 & 99.434 & 88.850 & 1223.90 \\
\hline s38584 & 1464 & s2 & 8 & 284 & 48.71 & 95.567 & 99.832 & 96.690 & 8719.52 \\
\hline i2c & 145 & 3 & 1 & 135 & 50.19 & 100.000 & 94.822 & 0.000 & 8.14 \\
\hline i2c & 145 & 3 & 2 & 135 & 50.19 & 100.000 & 96.062 & 23.936 & 116.21 \\
\hline i2c & 145 & 3 & 5 & 135 & 50.19 & 100.000 & 96.613 & 34.574 & 457.21 \\
\hline s13207 & 700 & 2 & 1 & 474 & 51.02 & 98.869 & 90.880 & 0.000 & 9.44 \\
\hline s13207 & 700 & 2 & 2 & 474 & 51.02 & 98.869 & 94.591 & 40.693 & 750.51 \\
\hline s13207 & 700 & 2 & 9 & 474 & 51.02 & 98.869 & 95.298 & 48.449 & 7042.33 \\
\hline i2c & 145 & s2 & 1 & 90 & 51.72 & 100.000 & 90.077 & 0.000 & 8.00 \\
\hline i2c & 145 & s2 & 2 & 90 & 51.72 & 100.000 & 96.031 & 60.000 & 119.09 \\
\hline i2c & 145 & s2 & 6 & 90 & 51.72 & 100.000 & 97.354 & 73.333 & 665.82 \\
\hline sasc & 132 & 3 & 1 & 66 & 51.97 & 100.000 & 86.746 & 0.000 & 5.17 \\
\hline sasc & 132 & 3 & 2 & 66 & 51.97 & 100.000 & 92.659 & 44.615 & 161.83 \\
\hline sasc & 132 & 3 & 4 & 66 & 51.97 & 100.000 & 93.026 & 47.385 & 502.33 \\
\hline spi & 274 & 3 & 1 & 1206 & 53.01 & 99.992 & 90.798 & 0.000 & 15.03 \\
\hline spi & 274 & 3 & 2 & 1206 & 53.01 & 99.992 & 92.946 & 23.343 & 276.91 \\
\hline spi & 274 & 3 & 25 & 1206 & 53.01 & 99.992 & 94.644 & 41.792 & 5770.38 \\
\hline b15 & 483 & s2 & 1 & 850 & 53.26 & 98.938 & 90.626 & 0.000 & 12.14 \\
\hline b15 & 483 & s2 & 2 & 850 & 53.26 & 98.938 & 96.943 & 67.391 & 295.02 \\
\hline b15 & 483 & s2 & 20 & 850 & 53.26 & 98.941 & 99.049 & 89.855 & 5475.68 \\
\hline
\end{tabular}

Column ntime shows the normalized runtime for the computation of $T_{1}, T_{2}, \ldots, T_{k}$. The normalized runtime is the runtime divided by the runtime for fault simulation of $T_{0}$. The runtime for $T_{1}$ includes the reordering of $T_{0}$ before it is truncated.

The following points can be seen from Tables 3 to 7 . With both values of $\mu$, and considering all or a subset of the gate-exhaustive faults, the size of $T_{1}$ as a percentage of $T_{0}$ varies significantly with the circuit. The gate-exhaustive fault efficiency of the truncated test set also varies with the circuit. Overall, Tables 3 to 7 show a wide range of truncated test sets. In several cases at the end 
Table 6. Experimental Results $55 \% \leq\left|T_{1}\right| /\left|T_{0}\right|<70 \%$

\begin{tabular}{|c|c|c|c|c|c|c|c|c|c|}
\hline circuit & inp & $\mu$ & $k$ & tests & \%tests & s.a. & g.exh & gap & ntime \\
\hline s9234 & 247 & s2 & 1 & 286 & 55.00 & 93.946 & 89.364 & 0.000 & 14.37 \\
\hline s9234 & 247 & s2 & 2 & 286 & 55.00 & 93.946 & 94.349 & 46.875 & 313.39 \\
\hline s9234 & 247 & s2 & 8 & 286 & 55.00 & 93.946 & 95.394 & 56.696 & 2089.03 \\
\hline wb_dma & 738 & 3 & 1 & 195 & 55.40 & 100.000 & 96.942 & 0.000 & 15.91 \\
\hline wb_dma & 738 & 3 & 2 & 195 & 55.40 & 100.000 & 97.968 & 33.543 & 822.42 \\
\hline wb_dma & 738 & 3 & 11 & 195 & 55.40 & 100.000 & 98.372 & 46.751 & 9747.42 \\
\hline tv80 & 372 & 2 & 1 & 1016 & 55.46 & 99.688 & 95.320 & 0.000 & 13.89 \\
\hline tv80 & 372 & 2 & 2 & 1016 & 55.46 & 99.692 & 96.958 & 34.992 & 196.87 \\
\hline tv80 & 372 & 2 & 35 & 1016 & 55.46 & 99.709 & 98.210 & 61.742 & 6512.72 \\
\hline systemcaes & 928 & 3 & 1 & 360 & 59.11 & 99.997 & 94.908 & 0.000 & 11.62 \\
\hline systemcaes & 928 & 3 & 2 & 360 & 59.11 & 99.997 & 95.683 & 15.231 & 511.46 \\
\hline systemcaes & 928 & 3 & 11 & 360 & 59.11 & 99.997 & 96.248 & 26.327 & 7023.50 \\
\hline usb_phy & 112 & 2 & 1 & 64 & 60.95 & 100.000 & 95.860 & 0.000 & 6.00 \\
\hline usb_phy & 112 & 2 & 2 & 64 & 60.95 & 100.000 & 98.318 & 59.375 & 122.00 \\
\hline usb_phy & 112 & 2 & 4 & 64 & 60.95 & 100.000 & 98.512 & 64.062 & 406.25 \\
\hline s5378 & 214 & s2 & 1 & 222 & 60.99 & 98.867 & 90.660 & 0.000 & 9.66 \\
\hline s5378 & 214 & $\mathrm{~s} 2$ & 2 & 222 & 60.99 & 98.867 & 98.319 & 82.000 & 189.65 \\
\hline s5378 & 214 & s2 & 7 & 222 & 60.99 & 98.867 & 99.626 & 96.000 & 1143.46 \\
\hline s13207 & 700 & s2 & 1 & 474 & 61.24 & 98.869 & 92.832 & 0.000 & 10.09 \\
\hline s13207 & 700 & $\mathrm{~s} 2$ & 2 & 474 & 61.24 & 98.869 & 98.755 & 82.632 & 904.42 \\
\hline s13207 & 700 & $\mathrm{~s} 2$ & 7 & 474 & 61.24 & 98.869 & 99.774 & 96.842 & 5691.81 \\
\hline b15 & 483 & 3 & 1 & 1275 & 61.56 & 98.938 & 96.101 & 0.000 & 14.88 \\
\hline b15 & 483 & 3 & 2 & 1275 & 61.56 & 98.941 & 97.529 & 36.636 & 433.02 \\
\hline b15 & 483 & 3 & 30 & 1275 & 61.56 & 98.941 & 98.262 & 55.416 & 12260.40 \\
\hline b14 & 280 & s2 & 1 & 708 & 63.38 & 95.966 & 90.087 & 0.000 & 10.85 \\
\hline b14 & 280 & s2 & 2 & 708 & 63.38 & 96.147 & 91.551 & 14.767 & 360.93 \\
\hline b14 & 280 & $\mathrm{~s} 2$ & 12 & 708 & 63.38 & 96.246 & 92.681 & 26.166 & 3860.31 \\
\hline spi & 274 & s2 & 1 & 806 & 64.64 & 99.992 & 88.636 & 0.000 & 12.96 \\
\hline spi & 274 & $\mathrm{~s} 2$ & 2 & 806 & 64.64 & 99.992 & 97.589 & 78.780 & 87.33 \\
\hline spi & 274 & $\mathrm{~s} 2$ & 7 & 806 & 64.64 & 99.992 & 99.889 & 99.024 & 452.95 \\
\hline des_area & 367 & 3 & 1 & 348 & 67.57 & 100.000 & 93.868 & 0.000 & 20.55 \\
\hline des_area & 367 & 3 & 2 & 348 & 67.57 & 100.000 & 98.360 & 73.254 & 420.62 \\
\hline des_area & 367 & 3 & 21 & 348 & 67.57 & 100.000 & 99.801 & 96.758 & 9237.82 \\
\hline s5378 & 214 & 3 & 1 & 333 & 67.68 & 98.867 & 94.819 & 0.000 & 10.72 \\
\hline s5378 & 214 & 3 & 2 & 333 & 67.68 & 98.867 & 97.511 & 51.952 & 222.10 \\
\hline s5378 & 214 & 3 & 12 & 333 & 67.68 & 98.867 & 98.849 & 77.778 & 2482.85 \\
\hline
\end{tabular}

of Table 7, no truncation is needed with the limit on the number of tests. These cases are included for completeness.

In most of the cases, the procedure described in this article is able to increase the gate-exhaustive fault efficiency of $T_{1}$ significantly. Even when the fault efficiency achieved by $T_{1}$ is high, the increase captured by the gap coverage is large.

The normalized runtime increases with the number of iterations. Per iteration, it is similar for circuits of different sizes with different gate-exhaustive fault efficiencies. This indicates that the procedure scales similar to a fault simulation procedure. 
Table 7. Experimental Results $\left|T_{1}\right| /\left|T_{0}\right| \geq 70 \%$

\begin{tabular}{l|c|cc|cc|cccc}
\hline circuit & inp & $\mu$ & $k$ & tests & \%tests & s.a. & g.exh & gap & ntime \\
\hline s13207 & 700 & 3 & 1 & 711 & 76.53 & 98.869 & 97.840 & 0.000 & 9.21 \\
s13207 & 700 & 3 & 2 & 711 & 76.53 & 98.869 & 98.934 & 50.655 & 982.02 \\
s13207 & 700 & 3 & 13 & 711 & 76.53 & 98.869 & 99.528 & 78.166 & 13141.73 \\
\hline tv80 & 372 & s2 & 1 & 1016 & 80.70 & 99.688 & 97.234 & 0.000 & 13.85 \\
tv80 & 372 & s2 & 2 & 1016 & 80.70 & 99.702 & 99.112 & 67.895 & 162.86 \\
tv80 & 372 & s2 & 11 & 1016 & 80.70 & 99.719 & 99.782 & 92.105 & 1503.57 \\
\hline tv80 & 372 & 3 & 1 & 1524 & 83.19 & 99.688 & 98.996 & 0.000 & 13.93 \\
tv80 & 372 & 3 & 2 & 1524 & 83.19 & 99.705 & 99.316 & 31.884 & 266.10 \\
tv80 & 372 & 3 & 12 & 1524 & 83.19 & 99.709 & 99.589 & 59.058 & 2907.29 \\
\hline usb_phy & 112 & s2 & 1 & 64 & 85.33 & 100.000 & 98.705 & 0.000 & 6.33 \\
usb_phy & 112 & s2 & 2 & 64 & 85.33 & 100.000 & 100.000 & 100.000 & 103.33 \\
\hline systemcdes & 320 & 2 & 1 & 156 & 89.14 & 100.000 & 99.646 & 0.000 & 18.80 \\
systemcdes & 320 & 2 & 2 & 156 & 89.14 & 100.000 & 100.000 & 100.000 & 280.84 \\
\hline aes_core & 788 & 2 & 1 & 416 & 89.46 & 100.000 & 99.741 & 0.000 & 28.41 \\
aes_core & 788 & 2 & 2 & 416 & 89.46 & 100.000 & 100.000 & 100.000 & 293.94 \\
\hline usb_phy & 112 & 3 & 1 & 96 & 91.43 & 100.000 & 99.612 & 0.000 & 8.00 \\
usb_phy & 112 & 3 & 2 & 96 & 91.43 & 100.000 & 99.935 & 83.333 & 199.67 \\
\hline aes_core & 788 & s2 & 1 & 416 & 95.41 & 100.000 & 99.928 & 0.000 & 19.65 \\
aes_core & 788 & s2 & 2 & 416 & 95.41 & 100.000 & 100.000 & 100.000 & 183.65 \\
\hline s35932 & 1763 & 2 & 1 & 40 & 100.00 & 89.781 & 100.000 & - & 5.69 \\
\hline s35932 & 1763 & 3 & 1 & 40 & 100.00 & 89.781 & 100.000 & - & 5.77 \\
\hline s35932 & 1763 & s2 & 1 & 39 & 100.00 & 89.781 & 100.000 & - & 6.81 \\
\hline systemcdes & 320 & 3 & 1 & 175 & 100.00 & 100.000 & 100.000 & - & 15.93 \\
\hline systemcdes & 320 & s2 & 1 & 150 & 100.00 & 100.000 & 100.000 & - & 16.16 \\
\hline aes_core & 788 & 3 & 1 & 465 & 100.00 & 100.000 & 100.000 & - & 28.33 \\
\hline
\end{tabular}

When the number of iterations is large, it is typically possible to terminate the procedure after a smaller number of iterations with a significantly reduced runtime and a small loss in fault efficiency. This can be seen from the increase in the fault efficiency achieved in iteration $k=2$ compared with the final iteration. To demonstrate this point more clearly, the results of all of the iterations for $s 38417$ with $\mu=2$ are shown in Figure 3 . There is a circle in Figure 3 for every iteration, showing the normalized runtime divided by 1000 on the horizontal axis, and the gate-exhaustive fault efficiency on the vertical axis. Figure 3 demonstrates that an earlier termination condition is possible with a small loss in fault efficiency.

\section{EXPERIMENTAL RESULTS FOR COMPRESSED LAUNCH-ON-SHIFT TESTS}

This section presents the results of applying the procedure for increasing the fault coverage of a truncated test set to benchmark circuits using compressed launch-on-shift tests for stuck-at, transition, single-cycle and two-cycle gate-exhaustive faults.

The set of single-cycle gate-exhaustive faults is the same as the one used in Section 5. To define two-cycle gate-exhaustive faults, the gate and input pattern from every single-cycle gateexhaustive fault is used for the second pattern of one or more faults. Every input pattern of the same gate that differs from the second pattern in the value of a single input is used as a first pattern. The resulting two-cycle gate-exhaustive fault is considered if the two input patterns yield different values on the output of the gate. 


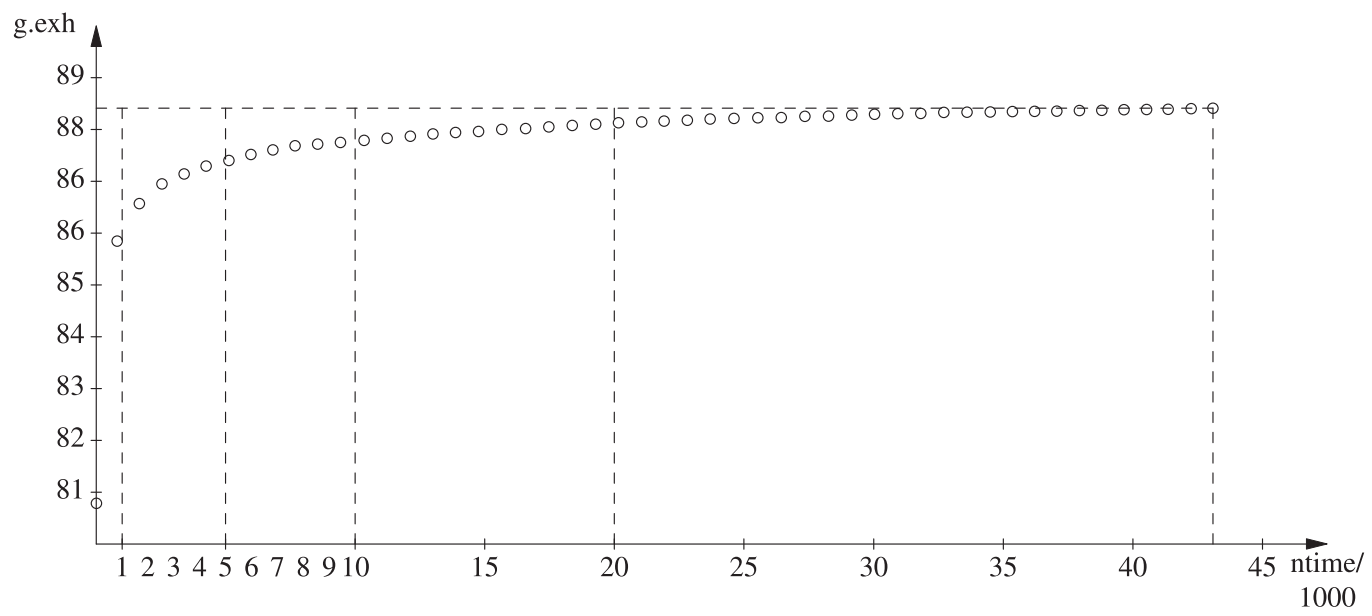

Fig. 3. Results for $s 38417$ with $\mu=2$.

The test set $T_{0}$ targets the four fault models. The detected faults are included in $D_{0}$. The remaining single-cycle and two-cycle gate-exhaustive faults are removed from consideration.

When $T_{0}$ is truncated, the test set $T_{1}$ detects all of the stuck-at and transition faults detected by $T_{0}$. A loss of fault coverage occurs for single-cycle and two-cycle gate-exhaustive faults. The number of tests in $T_{1}$ is determined using $\mu=2$, with $\theta_{0}$ being the number of tests in a compact test set for single stuck-at and transition faults.

When $T_{1}$ is modified to increase the set $D_{1}$ of faults that it detects, the procedure considers single-cycle and two-cycle gate-exhaustive faults together. Thus, it accepts a modification that detects more faults of one type and fewer of the other type if the overall number of detected faults is increased. To capture the overall effect, a combined fault efficiency is computed for gateexhaustive faults of both types.

The tests in $T_{0}$ are launch-on-shift tests that are compressed into seeds for an LFSR. To modify tests and increase their sets of detected faults, the procedure complements bits of the seeds that produce them. Thus, the number of bits that need to be considered is significantly smaller than the number of inputs and equal to the number of LFSR bits. Complementing bits of the compressed tests, instead of complementing bits of the applied tests, ensures that the tests remain applicable under the same test data compression approach.

The results are presented in Table 8. The format is similar to that of Tables 3 to 7. Column LFSR shows the number of LFSR bits used for compressed tests. The fault coverage metrics reported in Table 8 are the following. Column s.a. shows the stuck-at fault coverage. Column trans shows the transition fault coverage. Column g.exh 1 shows the single-cycle gate-exhaustive fault efficiency. Column g.exh2 shows the two-cycle gate-exhaustive fault efficiency. Column g.exh12 shows the combined single-cycle and two-cycle gate-exhaustive fault efficiency. Column gap measures the improvement in the gate-exhaustive fault efficiency considering both types of faults.

Table 8 shows improvements in the gap coverage that are similar to those in Tables 3 to 7.

To increase the gate-exhaustive fault efficiency considering both types of gate-exhaustive faults together, the procedure may allow the fault efficiency for single-cycle faults to decrease while increasing the fault efficiency for two-cycle faults. To avoid this effect, it is possible to consider the two fault types separately and require that the number of detected faults would not decrease for either one of them as compressed tests are modified. 
Table 8. Experimental Results for Four Fault Models and Compressed Tests

\begin{tabular}{|c|c|c|c|c|c|c|c|c|c|c|c|c|c|}
\hline circuit & inp & LFSR & $\mu$ & $k$ & tests & \%tests & s.a. & trans & g.exh1 & g.exh2 & g.exh12 & gap & ntime \\
\hline b14 & 280 & 128 & 2 & 1 & 978 & 42.65 & 96.263 & 95.390 & 92.381 & 88.240 & 90.486 & 0.000 & 7.24 \\
\hline b14 & 280 & 128 & 2 & 2 & 978 & 42.65 & 96.269 & 95.390 & 91.795 & 95.534 & 93.506 & 31.745 & 74.22 \\
\hline b14 & 280 & 128 & 2 & 25 & 978 & 42.65 & 96.269 & 95.390 & 91.828 & 99.289 & 95.243 & 50.000 & 1550.84 \\
\hline s15850 & 611 & 57 & 2 & 1 & 852 & 48.44 & 97.511 & 93.052 & 91.308 & 97.251 & 93.686 & 0.000 & 8.48 \\
\hline s15850 & 611 & 57 & 2 & 2 & 852 & 48.44 & 97.511 & 93.052 & 90.848 & 98.999 & 94.109 & 6.706 & 14.72 \\
\hline s15850 & 611 & 57 & 2 & 17 & 852 & 48.44 & 97.511 & 93.052 & 92.213 & 99.609 & 95.172 & 23.542 & 118.13 \\
\hline spi & 274 & 44 & 2 & 1 & 1468 & 50.73 & 99.992 & 98.112 & 92.154 & 77.677 & 87.450 & 0.000 & 15.06 \\
\hline spi & 274 & 44 & 2 & 2 & 1468 & 50.73 & 99.992 & 98.112 & 90.878 & 87.866 & 89.899 & 19.515 & 24.59 \\
\hline spi & 274 & 44 & 2 & 27 & 1468 & 50.73 & 99.992 & 98.112 & 92.307 & 93.155 & 92.582 & 40.896 & 257.26 \\
\hline s5378 & 214 & 36 & 2 & 1 & 630 & 53.16 & 98.867 & 93.022 & 98.520 & 74.765 & 91.173 & 0.000 & 11.41 \\
\hline s5378 & 214 & 36 & 2 & 2 & 630 & 53.16 & 98.867 & 93.022 & 98.208 & 77.515 & 91.808 & 7.195 & 20.06 \\
\hline s5378 & 214 & 36 & 2 & 22 & 630 & 53.16 & 98.867 & 93.022 & 98.894 & 84.267 & 94.370 & 36.220 & 192.40 \\
\hline s13207 & 700 & 47 & 2 & 1 & 1050 & 54.63 & 98.869 & 90.599 & 96.796 & 88.042 & 94.976 & 0.000 & 10.22 \\
\hline s13207 & 700 & 47 & 2 & 2 & 1050 & 54.63 & 98.869 & 90.599 & 96.863 & 90.364 & 95.512 & 10.666 & 17.04 \\
\hline s13207 & 700 & 47 & 2 & 21 & 1050 & 54.63 & 98.869 & 90.599 & 98.273 & 93.308 & 97.241 & 45.083 & 146.64 \\
\hline tv80 & 372 & 109 & 2 & 1 & 1702 & 57.81 & 99.723 & 95.612 & 98.849 & 84.954 & 93.156 & 0.000 & 11.78 \\
\hline tv80 & 372 & 109 & 2 & 2 & 1702 & 57.81 & 99.723 & 95.612 & 98.470 & 88.008 & 94.184 & 15.017 & 34.19 \\
\hline tv80 & 372 & 109 & 2 & 76 & 1702 & 57.81 & 99.730 & 95.633 & 98.820 & 94.805 & 97.175 & 58.718 & 1925.82 \\
\hline usb_phy & 112 & 18 & 2 & 1 & 182 & 58.90 & 100.000 & 97.395 & 100.000 & 86.567 & 94.291 & 0.000 & 8.20 \\
\hline usb_phy & 112 & 18 & 2 & 2 & 182 & 58.90 & 100.000 & 97.395 & 99.805 & 89.289 & 95.336 & 18.301 & 24.50 \\
\hline usb_phy & 112 & 18 & 2 & 11 & 182 & 58.90 & 100.000 & 97.395 & 99.870 & 94.118 & 97.425 & 54.902 & 159.20 \\
\hline $\mathrm{i} 2 \mathrm{c}$ & 145 & 43 & 2 & 1 & 304 & 62.94 & 100.000 & 96.247 & 98.923 & 84.002 & 93.292 & 0.000 & 10.28 \\
\hline $\mathrm{i} 2 \mathrm{c}$ & 145 & 43 & 2 & 2 & 304 & 62.94 & 100.000 & 96.247 & 97.790 & 90.474 & 95.029 & 25.897 & 41.02 \\
\hline $\mathrm{i} 2 \mathrm{c}$ & 145 & 43 & 2 & 13 & 304 & 62.94 & 100.000 & 96.270 & 98.011 & 95.807 & 97.179 & 57.949 & 364.21 \\
\hline s9234 & 247 & 75 & 2 & 1 & 710 & 65.80 & 93.946 & 87.736 & 95.770 & 99.312 & 96.936 & 0.000 & 15.25 \\
\hline s9234 & 247 & 75 & 2 & 2 & 710 & 65.80 & 93.946 & 87.736 & 95.685 & 99.656 & 96.992 & 1.847 & 41.63 \\
\hline s9234 & 247 & 75 & 2 & 6 & 710 & 65.80 & 93.946 & 87.752 & 95.721 & 99.853 & 97.081 & 4.749 & 152.35 \\
\hline sasc & 132 & 13 & 2 & 1 & 182 & 65.94 & 100.000 & 99.543 & 98.775 & 88.526 & 95.911 & 0.000 & 7.85 \\
\hline sasc & 132 & 13 & 2 & 2 & 182 & 65.94 & 100.000 & 99.543 & 98.530 & 91.053 & 96.440 & 12.950 & 18.54 \\
\hline sasc & 132 & 13 & 2 & 11 & 182 & 65.94 & 100.000 & 99.543 & 98.857 & 93.263 & 97.293 & 33.813 & 108.69 \\
\hline simple_spi & 146 & 38 & 2 & 1 & 248 & 68.32 & 100.000 & 97.906 & 94.135 & 94.602 & 94.245 & 0.000 & 7.59 \\
\hline simple_spi & 146 & 38 & 2 & 2 & 248 & 68.32 & 100.000 & 97.906 & 94.557 & 95.458 & 94.770 & 9.123 & 36.85 \\
\hline simple_spi & 146 & 38 & 2 & 15 & 248 & 68.32 & 100.000 & 97.906 & 95.535 & 97.258 & 95.941 & 29.474 & 373.48 \\
\hline $\mathrm{b} 20$ & 527 & 119 & 2 & 1 & 1328 & 71.09 & 95.675 & 94.194 & 98.591 & 99.336 & 98.877 & 0.000 & 15.10 \\
\hline b20 & 527 & 119 & 2 & 2 & 1328 & 71.09 & 95.677 & 94.196 & 98.532 & 99.711 & 98.985 & 9.607 & 47.82 \\
\hline b20 & 527 & 119 & 2 & 13 & 1328 & 71.09 & 95.693 & 94.215 & 98.609 & 99.894 & 99.103 & 20.087 & 388.14 \\
\hline systemcdes & 320 & 14 & 2 & 1 & 438 & 73.74 & 100.000 & 99.867 & 100.000 & 96.292 & 98.479 & 0.000 & 9.29 \\
\hline systemcdes & 320 & 14 & 2 & 2 & 438 & 73.74 & 100.000 & 99.867 & 99.978 & 96.387 & 98.505 & 1.717 & 12.34 \\
\hline systemcdes & 320 & 14 & 2 & 10 & 438 & 73.74 & 100.000 & 99.867 & 99.978 & 96.785 & 98.668 & 12.446 & 48.52 \\
\hline wb_dma & 738 & 47 & 2 & 1 & 536 & 75.49 & 100.000 & 98.496 & 99.434 & 91.216 & 96.720 & 0.000 & 9.65 \\
\hline wb_dma & 738 & 47 & 2 & 2 & 536 & 75.49 & 100.000 & 98.496 & 98.996 & 93.801 & 97.280 & 17.083 & 24.60 \\
\hline wb_dma & 738 & 47 & 2 & 28 & 536 & 75.49 & 100.000 & 98.526 & 99.241 & 96.450 & 98.319 & 48.752 & 377.57 \\
\hline b15 & 483 & 113 & 2 & 1 & 1564 & 75.78 & 98.915 & 96.289 & 98.976 & 98.866 & 98.941 & 0.000 & 12.94 \\
\hline b15 & 483 & 113 & 2 & 2 & 1564 & 75.78 & 98.915 & 96.297 & 98.899 & 99.351 & 99.044 & 9.718 & 46.39 \\
\hline b15 & 483 & 113 & 2 & 21 & 1564 & 75.78 & 98.918 & 96.311 & 98.956 & 99.832 & 99.236 & 27.887 & 858.86 \\
\hline aes_core & 788 & 28 & 2 & 1 & 2002 & 78.54 & 100.000 & 99.251 & 100.000 & 97.541 & 98.927 & 0.000 & 17.73 \\
\hline aes_core & 788 & 28 & 2 & 2 & 2002 & 78.54 & 100.000 & 99.251 & 100.000 & 97.646 & 98.973 & 4.269 & 20.15 \\
\hline aes_core & 788 & 28 & 2 & 38 & 2002 & 78.54 & 100.000 & 99.251 & 100.000 & 98.630 & 99.402 & 44.269 & 152.32 \\
\hline s38584 & 1464 & 98 & 2 & 1 & 1360 & 81.49 & 95.567 & 90.417 & 100.000 & 96.870 & 98.720 & 0.000 & 17.80 \\
\hline s38584 & 1464 & 98 & 2 & 2 & 1360 & 81.49 & 95.567 & 90.417 & 99.829 & 97.984 & 99.075 & 27.710 & 26.41 \\
\hline s38584 & 1464 & 98 & 2 & 43 & 1360 & 81.49 & 95.567 & 90.417 & 99.949 & 99.536 & 99.780 & 82.822 & 400.18 \\
\hline s38417 & 1664 & 111 & 2 & 1 & 2088 & 85.36 & 99.680 & 98.200 & 99.283 & 99.560 & 99.378 & 0.000 & 23.07 \\
\hline s38417 & 1664 & 111 & 2 & 2 & 2088 & 85.36 & 99.680 & 98.200 & 99.201 & 99.848 & 99.424 & 7.301 & 32.14 \\
\hline s38417 & 1664 & 111 & 2 & 25 & 2088 & 85.36 & 99.680 & 98.200 & 99.465 & 99.972 & 99.640 & 42.035 & 298.86 \\
\hline systemcaes & 928 & 29 & 2 & 1 & 750 & 91.35 & 99.997 & 99.519 & 99.771 & 98.996 & 99.501 & 0.000 & 12.97 \\
\hline systemcaes & 928 & 29 & 2 & 2 & 750 & 91.35 & 99.997 & 99.519 & 99.728 & 99.317 & 99.585 & 16.822 & 22.24 \\
\hline systemcaes & 928 & 29 & 2 & 17 & 750 & 91.35 & 99.997 & 99.519 & 99.875 & 99.525 & 99.753 & 50.467 & 179.52 \\
\hline des_area & 367 & 14 & 2 & 1 & 736 & 96.08 & 100.000 & 99.765 & 99.891 & 99.795 & 99.866 & 0.000 & 10.89 \\
\hline s35932 & 1763 & 13 & 2 & 1 & 240 & 97.56 & 89.781 & 88.666 & 100.000 & 99.980 & 99.990 & 0.000 & 14.07 \\
\hline s35932 & 1763 & 13 & 2 & 2 & 240 & 97.56 & 89.781 & 88.666 & 100.000 & 99.987 & 99.993 & 33.333 & 15.31 \\
\hline
\end{tabular}




\section{CONCLUDING REMARKS}

This article considered a test set $T_{1}$ obtained after truncating a larger test set $T_{0}$ to fit tester memory limits or satisfy constraints on test application time. Truncation causes the set of detected faults to decrease from $D_{0}$ to $D_{1}$. Without modifying the tests in $T_{0}$, the set $D_{1}$ is limited by the tests included in $T_{0}$. The procedure described in this article modifies the tests in $T_{1}$ to gain the detection of faults from $D_{0} \backslash D_{1}$, even at the cost of losing the detection of faults from $D_{1}$. The goal is to obtain a test set $T_{2}$ that detects a set of faults $D_{2}$ such that $\left|T_{2}\right|=\left|T_{1}\right|$ and $\left|D_{2}\right|>\left|D_{1}\right|$. In an iterative process, the procedure produces test sets $T_{k}$, for $k \geq 2$, such that $\left|T_{k}\right|=\left|T_{k-1}\right|$ and $\left|D_{k}\right|>\left|D_{k-1}\right|$. Experimental results were presented for gate-exhaustive faults in benchmark circuits to demonstrate the ability of the procedure to increase the fault coverage of a truncated test set.

\section{REFERENCES}

[1] A. Sreedhar, A. Sanyal, and S. Kundu. 2008. On modeling and testing of lithography related open faults in Nano-CMOS circuits. In Proc. Design, Autom. \& Test in Europe Conf. ACM, 616-621.

[2] M. O. Simsir, A. Bhoj, and N. K. Jha. 2010. Fault modeling for FinFET circuits. In Proc. Intl. Symp. on Nanoscale Architectures. 41-46.

[3] J. Zha, X. Cui, and C. L. Lee. 2012. Modeling and testing of interference faults in the nano NAND flash memory. In Proc. Design, Automation \& Test in Europe Conf. IEEE, 527-531.

[4] S.-Y. Huang, Y.-H. Lin, K.-H. Tsai, W.-T. Cheng, S. Sunter, Y.-F Chou, and D.-M. Kwai. 2012. Small delay testing for TSVs in 3-D ICs. In Proc. Design Automation Conf. 1031-1036.

[5] S. Di Carlo, G. Gambardella, P. Prinetto, D. Rolfo, and P. Trotta. 2015. SATTA: A self-adaptive temperature-based TDF awareness methodology for dynamically reconfigurable FPGAs. In ACM Trans. on Reconfigurable Technology and Systems 8, 1 (2015), Article 1, 1-22.

[6] H. G. Mohammadi, P.-E. Gaillardon, and G. De Micheli. 2015. Fault modeling in controllable polarity silicon nanowire circuits. In Proc. Design, Automation \& Test in Europe Conf. IEEE, 453-458.

[7] C.-Y. Hsieh, C.-H. Wu, C.-H. Huang, H.-S. Goan, and J. C. M. Li. 2020. Realistic fault models and fault simulation for quantum dot quantum circuits. In Proc. Design Automation Conf. IEEE, 46, 1-6.

[8] D. Kim, M. E. Amyeen, S. Venkataraman, I. Pomeranz, S. Basumallick, and B. Landau. 2007. Testing for systematic defects based on DFM guidelines. In Proc. Intl. Test Conf. IEEE, 1-10.

[9] A. Sinha, S. Pandey, A. Singhal, A. Sanyal, and A. Schmaltz. 2017. DFM-aware fault model and ATPG for intra-cell and inter-cell defects. In Proc. Intl. Test Conf. IEEE, 1-10.

[10] F. Hapke and J. Schloeffel. 2012. Introduction to the defect-oriented cell-aware test methodology for significant reduction of DPPM rates. In Proc. European Test Symp. 1-6.

[11] F. Yang, S. Chakravarty, A. Gunda, N. Wu, and J. Ning. 2014. Silicon evaluation of cell-aware ATPG tests and small delay tests. In Proc. Asian Test Symp. IEEE, 101-106.

[12] A. D. Singh. 2016. Cell aware and stuck-open tests. In Proc. European Test Symp. IEEE, 1-6.

[13] Y.-H. Huang, C.-H. Lu, T.-W. Wu, Y.-T. Nien, Y.-Y. Chen, M. Wu, Ji.-N. Lee, and M. C.-T. Chao. 2017. Methodology of generating dual-cell-aware tests. In Proc. VLSI Test Symp. IEEE, 1-6.

[14] S. P. Dixit, D. D. Vora, and K. Peng. 2018. Challenges in cell-aware test. In Proc. European Test Symp. 1-6.

[15] E. J. McCluskey. 1993. Quality and single-stuck faults. In Proc. Intl. Test Conf. IEEE, 597.

[16] R. Guo, S. Mitra, E. Amyeen, J. Lee, S. Sivaraj, and S. Venkataraman. 2006. Evaluation of test metrics: Stuck-at, bridge coverage estimate and gate exhaustive. In Proc. VLSI Test Symp. IEEE, 66-71.

[17] A. Jas, S. Natarajan, and S. Patil. 2007. The region-exhaustive fault model. In Proc. Asian Test Symp. 13-18.

[18] I. Pomeranz. 2019. Iterative test generation for gate-exhaustive faults to cover the sites of undetectable target faults. In Proc. Intl. Test Conf. Paper 1.3, IEEE, 1-7.

[19] I. Pomeranz. 2021. Maximal independent fault set for gate-exhaustive faults. IEEE Trans. on Computer-Aided Design 40, 3 (2021), 598-602.

[20] P. Goel and B. C. Rosales. 1979. Test generation and dynamic compaction of tests. In Proc. Test Conf. IEEE, 189-192.

[21] S. Kajihara, I. Pomeranz, K. Kinoshita, and S. M. Reddy. 1995. Cost-effective generation of minimal test sets for stuck-at faults in combinational logic circuits. In IEEE Trans. on Computer-Aided Design. IEEE, 1496-1504.

[22] D. Xiang, J. Li, K. Chakrabarty, and X. Lin. 2013. Test compaction for small-delay defects using an effective path selection scheme. ACM Trans. on Design Automation 18, 3 (2013), Article 44, 1-23.

[23] D. Xiang, K. Shen, B. B. Bhattacharya, X. Wen, and X. Lin. 2016. Thermal-aware small-delay defect testing in integrated circuits for mitigating overkill. IEEE Trans. on Computer-Aided Design 35, 3 (2016), 499-512. 
[24] W. Jiang and B. Vinnakota. 1999. Defect-oriented test scheduling. In Proc. VLSI Test Symp. IEEE, 433-438.

[25] X. Lin, J. Rajski, I. Pomeranz, and S. M. Reddy. 2001. On static test compaction and test pattern ordering for scan designs. In Proc. Intl. Test Conf. IEEE, 1088-1097.

[26] L. N. Reddy, I. Pomeranz, and S. M. Reddy. 1992. COMPACTEST-II: A method to generate compact two-pattern test sets for combinational logic circuits. In Proc. Intl. Conf. on Computer-Aided Design. IEEE, 568-574.

[27] R. Desineni, K. N. Dwarkanath, and R. D. Blanton. 2000. Universal test generation using fault tuples. In Proc. Intl. Test Conf. IEEE, 812-819.

[28] S. M. Reddy, G. Chen, J. Rajski, I. Pomeranz, P. Engelke, and B. Becker. 2005. A unified fault model and test generation procedure for interconnect opens and bridges. In Proc. Europ. Test Symp. IEEE, 22-27.

[29] S. Alampally, R. T. Venkatesh, P. Shanmugasundaram, R. A. Parekhji, and V. D. Agrawal. 2011. An efficient test data reduction technique through dynamic pattern mixing across multiple fault models. In Proc. VLSI Test Symp. IEEE, 285-290.

[30] C.-H. Wu and K.-J. Lee. 2016. Transformation of multiple fault models to a unified model for ATPG efficiency enhancement. In Proc. Intl. Test Conf. IEEE, 1-10.

Received July 2021; revised October 2021; accepted December 2021 